\title{
The puzzle of the proton radius
}

\author{
A K F Val Baker \\ Torus Tech LLC. San Clemente, California, 92673, USA \\ Hawaii Institute for Unified Physics, Kailua Kona, Hawaii, 96740, USA \\ Email: amira@hiup.org
}

\begin{abstract}
The radius of the proton is a topic of debate and has yet to be confirmed. Measurements from different techniques yield conflicting values, with some experimental methods yielding a value in disagreement with the standard model. This review article outlines the current status of the experimental results and summarizes the possible solutions to this puzzle.
\end{abstract}

Keywords: nucleonic parameters: radius - particle physics: proton - fundamental physics: standard model

\section{Introduction}

To understand the workings of the Universe, from the very big to the very small, we need to observe and measure the fundamental characteristics of the material world. Advances in technology enables measurements of greater accuracy and thus either confirms theories or leads us in new, and sometimes unknown, directions.

One of the most important characteristics of the objects we study is their spatial extent. However, when dealing with subatomic particles, our ability to directly measure the size is limited by the instruments we use. For example, techniques such as scanning probe microscopy, although achieving subatomic resolutions on the order of $\sim 0.1 \mathrm{~nm}$ [1], are unable to reveal the spatial extent of subatomic particles. We therefore rely on more indirect methods which instead of measuring the object in co-ordinate space measures the distribution of changes of momentum. This distribution is called the form factor which, based on theoretical assumptions, compensates for irregularity in the shape of an object and is essentially a Fourier transform. For example, the nucleon electromagnetic form factors describe the spatial distributions of electric charge and current inside the nucleon, which for the non-relativistic case is given in terms of the volume integral $F\left(q^{2}\right)=\int \rho(\vec{r}) e^{i \vec{q} \cdot \vec{r}} d^{3} \vec{r}[2]$.

The radius of an oscillating electrostatic field, such as a proton, defines an effective charge boundary in that region of space. This is typically referred to as a 'charge radius' and is described by its electric form factor. The standard approach to determining this radius thus relies on indirect measurements of the energy interaction at the charge surface boundary. Measurements of this energy interaction are typically done utilizing electron-proton scattering and/or hydrogen spectroscopy methods. Both these methods have consistently yielded similar results, where the latest 2014 CODATA value, $r_{p}=0.8751(61) f m$, is based on a leastsquares approximation between both methods [3]. However, spectroscopic measurements of the Lamb shift were also made utilizing muonic hydrogen as oppose to electronic hydrogen and yielded much smaller values than that of the combined electronic methods as given by CODATA. The first measurement of muonic 
hydrogen was made in 2010 and yielded a value of $r_{p}=0.84184(67) \mathrm{fm}$ [4], differing from the 2010 CODATA value,

$r_{p}=0.8775(51) \mathrm{fm}$, by $7 \sigma$ [5]. These measurements were repeated in 2013 and found a more precise value of $r_{p}=0.84087(39) \mathrm{fm}$ [6], differing from the 2010 CODATA value by $7.2 \sigma$.

The question is, why are these different methods (electronic and muonic) yielding different results? This is a problem that the physics community have been trying to reconcile, and the failure to understand it is known as the proton radius puzzle.

Due to the high correlation between the proton radius and the Rydberg constant, the same spectroscopic experiments are used to determine both values, thus any change in the charge radius of the proton also implies a change in the Rydberg constant. The value for the Rydberg constant is known with a relative uncertainty of $1.9 \times 10^{-12}$ and is one of the most accurate fundamental constants to date. Therefore, the electrical size of the proton, its charge radius, is now a limitation $(4-7 \sigma)$ to the accuracy of the Rydberg constant found from this comparison of theory [7] [8] [9].

In 2016 this discrepancy became even more significant when a measurement of the Lamb shift was made utilizing muonic deuterium, as oppose to electronic hydrogen, and yielded a value for the charge radius of deuteron of $r_{d}=2.12562(78) \mathrm{fm}$ [10]. This value found from muonic deuterium is smaller than the 2010 CODATA value of $r_{d}=2.1424(21) f m$ by $7.5 \sigma \quad$ [5] and smaller than the value of $r_{d}=2.1415(45) \mathrm{fm}$ found by electronic deuterium spectroscopy techniques by $3.5 \sigma$ [11]. This smaller radius for deuteron also yields a smaller value for the proton radius, $r_{p}=0.8356(20) \mathrm{fm}$, similar to that found from muonic hydrogen [10].

These results suggest that spectroscopic methods yield smaller values than those found from scattering techniques, with muonic spectroscopy measurements yielding the smallest values.

However, new experiments measuring the $2 S-2 P$ Lamb shift in electronic hydrogen found a significantly smaller value, $\quad r_{p}=0.833(10) \mathrm{fm}$, more in agreement with that found by muonic hydrogen and muonic deuterium [12]. Their approach claims to utilize special techniques to reduce quantum interference, from which they conclude yields a more precise and accurate result. If their result and the previous muonic measurements are correct, then what does this tell us about previous electronic measurements and quantum interference correction techniques.

Furthermore, in another recent study carried out at the Jefferson Laboratory, Virginia, USA, the measurement of the proton radius was made utilizing high precision electron-proton scattering techniques, yielding a value of $r_{p}=0.831(19) \mathrm{fm}$ [13]. This value is $3.2 \sigma$ smaller than the most recent high-precision electron-proton scattering measurement of $r_{p}=0.879(15) \mathrm{fm}$ [14] and 2.7 $\sigma$ smaller than the average of all electron-proton scattering measurements [3]. Remarkably, it is in a significant agreement with both the muonic hydrogen spectroscopy measurements and the latest electronic hydrogen measurements. So, it could be that the proton radius puzzle is not a puzzle anymore.

In this paper we will outline the two methods for measuring and determining the proton radius and discuss the possible sources of error or resolutions to the proton radius puzzle. 


\section{Measuring the radius of the proton}

\subsection{Electron scattering experiments}

Electron scattering was first applied by Robert McAllister and Robert Hofstadter in the 1950's as a technique for measuring the charge distribution of the proton, and to this day provides one of the most powerful tools to investigate nucleonic structure [15] [16].

Measurements of the proton radius are typically done using elastic electron-proton scattering, in which a beam of electrons is fired at a proton source (i.e. liquid hydrogen) and the scattering angles are measured. The scattering probability can be calculated as a function of proton radius, and thus the proton radius can be found by measuring the scattering angle.

In the case of electron-proton scattering, the differential scattering cross section $d \sigma / d \Omega$ of the scattered electrons can be described by the Mott scattering formula. This is an extension of low-energy Coulomb scattering (i.e. Rutherford scattering), which includes the effects of relativistic energies and spin-coupling inelastic Coulomb scattering, and is given as,

$$
\left.\frac{d \sigma}{d \Omega}\right|_{M o t t}=\frac{4 \alpha^{2} \cos ^{2}(\theta / 2)}{Q^{4}} \frac{E^{\prime 3}}{E}
$$

where $\sigma$ is the interaction cross section, $\Omega$ is the solid angle, $\alpha$ is the fine structure constant, $\theta$ is the scattering angle, $E$ is the incoming electron lab energy, $E^{\prime}$ is the outgoing electron lab energy and $Q^{2}$ is the four-momentum transfer.

However, for an extended target, which is the assumed case for a proton, the differential scattering cross section has to be modified by multiplying by the square of the form factor $F\left(Q^{2}\right)$. As well, since the charge distribution is not static due to recoil effects, and the magnetic and electric distributions will be independently effected by the spin, the form factors are separated for electric $G_{E}\left(Q^{2}\right)$ and magnetic $G_{M}\left(Q^{2}\right)$ distributions.

The multiplicative factor is therefore a function of both the magnetic and electric form factors and the solution is given as first order from perturbation theory, known as the Rosenbluth formula [17],

$$
\frac{d \sigma}{d \Omega}\left|=\frac{d \sigma}{d \Omega}\right|_{\text {Mott }} \times \frac{1}{(1+\tau)}\left(G_{E}^{2}\left(Q^{2}\right)+\frac{\tau}{\varepsilon} G_{M}^{2}\left(Q^{2}\right)\right)
$$

where $\tau=\frac{Q^{2}}{4 M^{2}}$,

$\varepsilon=\left[1+2(1+\tau) \tan ^{2}(\theta / 2)\right]^{-1}$ is the

kinematic factor and $G_{E}=F_{D}-\frac{Q^{2}}{4 M^{2}} F_{P}$ and $G_{M}=F_{D}+F_{P}$ are the electric and magnetic form factors in terms of the Dirac and Pauli form factors $F_{D}$ and $F_{P}$, respectively.

Similarly, the form factors can be determined by measuring the differential cross section of a variety of $Q^{2}$ and scattering angles $\theta$. The electric and magnetic radius can thus be found as the slope of the proton form factor at zero momentum transfer,

$$
\left\langle r_{E / M}^{2}\right\rangle=-\left.\frac{6 \hbar^{2}}{G_{E / M}(0)} \frac{d G_{E / M}\left(Q^{2}\right)}{d Q^{2}}\right|_{Q^{2}=0}
$$

The precision to which the radius is determined is therefore dependent on the data available at low $Q^{2}$ and the associated uncertainties. Recent work [14] utilising the Mainz Microtron (MAMI) located at the Johannes Gutenberg University in Mainz, Germany, successfully performed high-precision 
measurements and significantly expanded the data set at low $Q^{2}$. These results were included in the global examination of elastic electron-proton scattering data as given by Arrington and Sick [18] and Bernauer and Distler [3]. The latest results recommended by CODATA 2014 are found as the weighted mean between these two values, $r_{p}=0.880$ (11) $f m$ as given by Bernauer and Distler [3] and $r_{p}=0.879(11) \mathrm{fm}$ as given by Arrington and Sick [18], yielding a value of $r_{p}=0.879(13) \mathrm{fm}$.

\subsection{Hydrogen spectroscopy}

Another technique used to measure the proton radius is that of hydrogen spectroscopy, which again relies on indirect measurements of the energy interaction at the charge surface boundary between the electron and the proton. High precision spectroscopy of hydrogen reveals the transition frequencies of a multitude of spectral lines, from which a combination of at least two measurements can reveal the proton radius and the Rydberg constant simultaneously.

These transition frequencies or energy shifts can successfully be described in terms of the proton radius, $r_{p}$ by extensions to the Dirac equation that account for quantum corrections. For example, the Dirac equation can be given as,

$$
E_{n l j}=R_{\infty}\left(-\frac{1}{n^{2}}+f_{n l j}\left(\alpha, \frac{m_{e}}{m_{p}}, \ldots\right)+\delta_{l 0} \frac{C_{N S}}{n^{3}} r_{p}^{2}\right)
$$

where $n, l$ and $j$ are the principle, orbital and total angular momentum quantum numbers and $R_{\infty}=\frac{m_{e} \alpha^{2} c}{2 h}$ is the Rydberg constant.
The first term defines the fundamental structure of hydrogen as a function of its principle energy level whereas the second and third terms account for quantum corrections (i.e. relativistic recoil, vacuum polarization, nuclear finite-size and nuclear motion). Specifically, the second term accounts for relativistic corrections as well as contributions coming from the interactions of the bound-state system with the quantum vacuum and is given as a function in terms of the fine structure constant, $\alpha$ and the electron-to-proton mass ratio, $\mu=m_{e} / m_{p}$. Whereas the third term accounts for corrections originating from the nuclear finite-size.

The nuclear finite-size contributions are the second largest contribution to the splitting of energy levels, with the most significant contributing factor being the vacuum polarizations. Since the vacuum polarizations are calculated to arbitrary accuracy, and the remaining contributions are fairly accurately calculated, the nuclear finite-size effects remain the most uncertain component. Considerable effort has therefore been put into calculating these effects. However, numerical calculations for the finite-size effects have yielded varying results [19] suggesting a perturbative approach where the finite-size shifts in energy are computed utilizing form factors. Low lying $S$ states are the most sensitive to nuclear charge distributions, thus small S-state level energy shifts such as the Lamb shift provide precise constraints on nuclear charge distributions [20] [21] [22] [23] [24] [25].

The Lamb shift is the difference in energy between the two most tightly bounded exited states $\left(2 S_{1 / 2}\right.$ and $\left.2 P_{1 / 2}\right)$ due to interactions with the vacuum energy fluctuations. It is typically measured by exciting the $2 P_{1 / 2}$ into the $2 P_{3 / 2}$ state and detecting the subsequent photon decay to 
the $1 S_{1 / 2}$ state in coincidence with the laser pulse.

The latest 2014 CODATA value for the radius of the proton from spectroscopy measurements, $\quad r_{p}=0.8759(77) \mathrm{fm}, \quad$ is obtained from a combination of 24 transition frequency measurements in hydrogen and deuterium. However, although many of the individual measurements are in agreement with both the 2010 and 2013 muonic measurements the average is in a $4 \sigma$ disagreement. Therefore the 2014 CODATA value for the radius of the proton does not include the muonic measurements, instead making a least squares approximation between both the electronic methods [3].

Recent measurements in agreement with the muonic measurements have now brought back into question whether or not the muonic measurements should be included in the CODATA values [26] [12].

To increase accuracy and reduce corrections the Garching $1 S-2 S$ apparatus was utilized as a well-controlled cryogenic source of $5.8 \mathrm{~K}$ cold $2 \mathrm{~S}$ atoms. This reduced the thermal velocity of the atoms by a factor of 10 allowing successful measurement of the $2 S-4 P$ transition, yielding simultaneously a value for the proton radius of, $r_{p}=0.8335(95) \mathrm{fm}$ and a value for the Rydberg constant of $R_{\infty}=10973731.568076(96) m^{-1}$ [26]. This value for the Rydberg constant is in a $4 \sigma$ agreement with the latest 2018 CODATA value of $R_{\infty}=10973731.568160(21) \mathrm{m}^{-1}$.

Additionally, corrections due to quantum interference from neighboring atomic resonances were also considered. Such effects, although previously thought to be negligible [27] can cause deviations from a perfect Lorentzian line profile and are thus typically corrected utilizing numerical simulations [28] [29]. This requires highly accurate characterization of the experimental geometry which can result in extraneous errors, therefore, to eliminate any line shifts from quantum interference a simple line shape model was used.

These results were supported by recent experiments measuring the $2 S-2 P$ Lamb shift in electronic hydrogen, which also took significant steps to reduce quantum interference and improve the experimental precision. Utilizing a special technique specifically developed for this measurement, known as a 'frequencyoffset separated-oscillatory-field technique', a precise measurement of $r_{p}=0.833(10) \mathrm{fm}$ was found [12]. Both these latest results [26] [12] are within a $1 \sigma$ agreement with that obtained from the muonic Lamb shift measurements of

$r_{p}=0.84184(67) f m[4]$ and $r_{p}=0.84087(39) f m[6]$.

\section{Possible resolutions}

How can we solve this puzzle? First, we need to understand the difference between 'normal' electronic hydrogen and 'exotic' muonic hydrogen. The former is the most basic atom consisting of a single proton nucleus and a single electron, whereas the latter also consists of a single proton, but instead of an electron it hosts its heavier brother from the lepton family, the muon. See Fig 1.

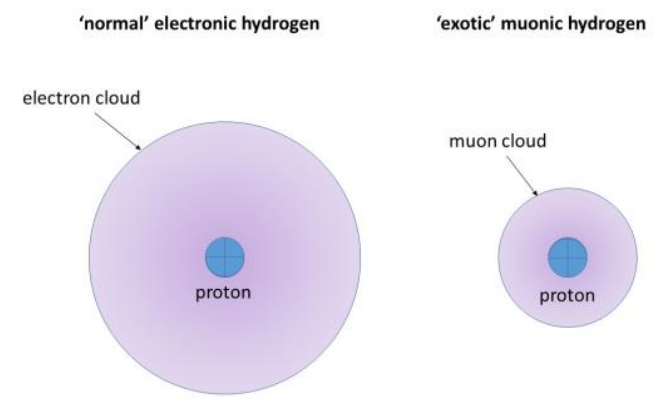

Figure 1: diagram (not to scale) to illustrate the difference between 'normal' electronic hydrogen and muonic hydrogen. 
The accuracy of the muonic measurements is enabled by the fact that the muons orbit is $\sim 200$ times smaller than the electrons orbit and therefore the sensitivities of the energy levels to the effective proton boundary are much greater. The proton radius is therefore determined to much greater accuracy with muonic hydrogen as oppose to electronic hydrogen. However, in the case of electronic hydrogen the value for $R_{\infty}$ is found consistently from multiple frequency measurements of varying degrees of accuracy and as well are in good agreement with theory [30] [7]. This agreement between theory and experiment strongly suggests that the value and its high degree of accuracy are correct and thus any disagreement with the muonic measurement remains a puzzle.

Possible causes for the discrepancy point to either experimental or theoretical error, and/or the possibility of new physics beyond the standard model. For example, it could be that the quantum electrodynamics (QED) calculations that account for contributions to the Lamb shift are inaccurate. In addition, it could be that one of the most well-determined physical constants, the Rydberg constant, needs to be corrected. Another possibility is that the electron and muon interact differently with the proton, but this would violate the idea, known as lepton universality, that electrons, tau leptons and muons all behave the same and are produced at the same rate [7] [8] [9]. Note, recent experiments at the Large Hadron Collider show the first clues that this may not be the case and that in fact lepton universality violation is the norm [31].

Maybe it's a combination of all these things as well as the need to further explore new ideas in physics.

The leading theory in new physics beyond the standard model, in the quest for quantum gravity, is loop quantum gravity. Other approaches include MOND's solution to dark matter and string theory's $\mathrm{N}$-dimensional approach to a quantum description of gravity. However, none of these approaches have yet offered a solution to the proton radius puzzle. Another more alternative approach is that of the generalized holographic approach, in which a solution for the mass of the proton is based on volume considerations of the holographic solution to an entropic bound [32] [33]. For an overview on the history and development of the holographic principle and a summary of the generalised holographic approach see references [34] [35].

Essentially, the generalised holographic solution describes a quantised solution to gravity in terms of the Planck granular structure of space time. When these considerations are applied at the nucleonic scale a precise value for the mass, $m_{p}$ and charge radius, $r_{p}$, of a proton can be given as,

$$
\begin{aligned}
& m_{p}=2 \frac{\eta}{R} m_{\ell}=2 \phi m_{\ell} \\
& r_{p}=4 \ell \frac{m_{\ell}}{m_{p}}=0.841236(28) \mathrm{fm}
\end{aligned}
$$

where $\phi=\eta / R$ is a fundamental holographic ratio defining the relationship between the surface entropy $\eta$ and the volume entropy $R$. Note the value used for the proton mass when determining the proton radius is the 2018 CODATA value $m_{p}=1.67262192369(51) \times 10^{-27} \mathrm{~kg}$.

Significantly, this value for the radius of the proton is found within an $1 \sigma$ agreement with the latest muonic measurements of the charge radius of the proton [4] [6], relative to a $7 \sigma$ variance in the standard approach [3].

Furthermore, the mass of the proton is determined experimentally, utilizing penning trap devices, with no quantum corrections and a relative standard uncertainty of $3.1 \times 10^{-10}$. 


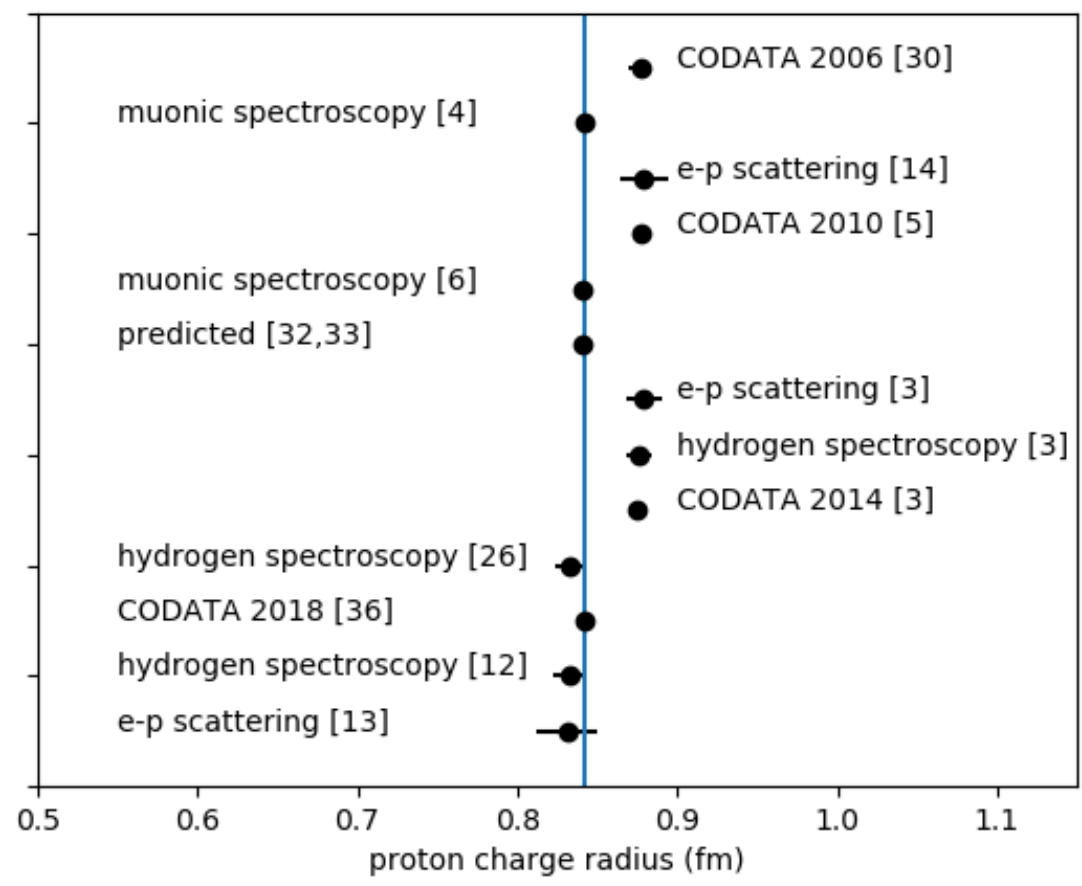

Figure 2: The proton charge radius as extracted from e-p scattering, hydrogen spectroscopy, muonic spectroscopy, the 2006, 2010, 2014 and 2018 CODATA value, as well as the value predicted by theory. Note the references are shown in brackets for each case. The vertical blue line indicates the 2018 CODATA value.

The increasing level of accuracy in electronic measurements is reflected in the latest 2018 CODATA value, $r_{p}=0.8414(19) \mathrm{fm}$ [36]. From Fig. 2 we can see that the latest results, along with the prediction by the generalised holographic approach, all point to the muonic measurements not only being more precise but also more accurate.

\section{Summary}

It seems the problem is not the way the muon interacts with the field. Instead it is a combination of the quantum interference corrections and the precision and accuracy of the measuring techniques. When highly accurate measurements are taken, we get values from both electron-proton scattering and hydrogen spectroscopy in agreement with the earlier measurements of muonic hydrogen. Remarkably the predicted radius from the generalized holographic solution is in very good agreement. It therefore seems there is no puzzle and along with the accurate measurements we find a theoretically predicted radius from first principles with no free parameters and no quantum corrections.

Acknowledgements Thanks to Dr Ines Urdaneta for her useful comments and discussions.

\section{References}

[1] G. Binnig, F. Quate and C. Gerber, "Atomic Force Microscope," Phys. Rev. Letts., vol. 56, no. 9, pp. 930-934, 1986.

[2] R. Wilson, "Form Factors of Elementary Particles," Physics Today, vol. 22, no. 1, pp. 47-53, 1969.

[3] P. J. Mohr, D. B. Newell and B. N. Taylor, "CODATA Recommended Values of the Fundamental Physical Constants: 2014," Rev. Mod. Phys., vol. 88, no. 3, 2016.

[4] R. Pohl, A. Antognini, F. Nez and e. al., "The size of the proton," Nature Letts., vol. 466, pp. 213-217, 2010.

[5] P. J. Mohr, B. N. Taylor and D. B. Newell, "CODATA recommended values of the fundamental physical constants: 2010," Rev. Mod. Phys, vol. 84, no. 4, 2012.

[6] A. Antognini, F. Nez and K. Schuhmann, "Proton Structure from the Measurement of 2S-2P Transition Frequencies of Muonic Hydrogen," Science, vol. 339, pp. 417-420, 2013.

[7] F. Nez, A. Antognini, F. D. Amaro, F. Biraben, J. M. R. Cardoso, D. Covita, A. Dax, 
S. Dhawan, A. Giesen, T. Graf, T. W. Hansch, P. Indelicato, C. -Y. Kao, P. E. Knowles, E. L. Bigot, Y. -W. Li, L. Ludhova, C. M. B. Monteiro, F. Mulhauser, T. Nebel, T. Nebel, P. Rabinowitz, J. M. F. Dos Santos, L. Schaller, K. Schuhmann, C. Schwob, D. Taqqu, F. Ko, F. Kottmann and R. Pohl, "Is the proton radius a player in the redefinition of the International System of Units?," Phil. Trans. R. Soc. A (2011) 369, 4064-4077, vol. 369, p. 4064-4077, 2011.

[8] R. Pohl, R. Gilman, G. A. Miller and K. Pachucki, "Muonic hydrogen and the proton radius puzzle," Annu. Rev. Nucl. Part. Sci., vol. 63, pp. 175-204, 2013.

[9] C. E. Carlson, "The Proton Radius Puzzle," Prog. Part. Nucl. Phys., vol. 82, pp. 59-77, 2015.

[10] R. Pohl, F. Nez, L. M. Fernandes and e. al., "Laser spectroscopy of muonic deuterium," Science, vol. 353, no. 6300, pp. 669-673, 2016.

[11] R. Pohl, F. Nez, T. Udem, A. Antognini, A. Beyer, H. Fleurbaey, A. Grinin, T. W. Hänsch, L. Julien, F. Kottmann, J. J. Krauth, L. Maisenbacher, A. Matveev and F. Biraben, "Deuteron charge radius and Rydberg constant from spectroscopy data in atomic deuterium," Metrologia, vol. 54, no. 2, 2017.

[12] N. Bezginov, T. Valdez, M. Horbatsch, A. Marsman, A. C. Vutha and E. A. Hessels, "A measurement of the atomic hydrogen Lamb shift and the proton charge radius," Science, vol. 365, pp. 1007-1012, 2019.

[13] W. Xiong, A. Gasparian, H. Gao, D. Dutta, M. Khandaker, N. Liyanage, E. Pasyuk, C. Peng, X. Bai, L. Ye, K. Gnanvo, C. Gu, M. Levillain, X. Yan, D. W. Higinbotham, M. Meziane, Z. Ye, K. Adhikari, B. Aljawrneh, H. Bhatt, D. Bhetuwal, J. Brock, V. Burkert, C. Carlin, A. Deur, D. Di, J. Dunne, P. Ekanayaka, L. El-Fassi, B. Emmich, L. Gan, O. Glamazdin, M. L. Kabir, A. Karki, C. Keith, S. Kowalski, V. Lagerquist, I. Larin, T. Liu, A. Liyanage, J. Maxwell, D. Meekins, S. J. Nazeer, V. Nelyubin, H. Nguyen, R. Pedroni, C. Perdrisat, J. Pierce, V. Punjabi, M. Shabestari, A. Shahinyan, R. Silwal, S. Stepanyan, A. Subedi, V. V. Tarasov, N. Ton, Y. Zhang and Z. W. Zhao, "A small proton charge radius from," Nature, vol. 575, pp. 147-151, 2019.

[14] J. C. Bernauer, P. Achenbach, C. Ayerbe Gayoso, R. Bohm, D. Bosnar, L. Debenjak, M. O. Distler, L. Doria, A. Esser, H. Fonvieille, J. M. Friedrich, J. Friedrich, M. Gomez Rodriguez de la Paz, M. Makek, H. Merkel, D. G. Middleton, U. Muller, L. Nungesser, J.
Pochodzalla, M. Potokar, S. Sanchez Majos, B. S. Schlimme, S. Sirca, T. Walcher and M. Weinriefer, "High-Precision Determination of the Electric and Magnetic Form Factors of the Proton," Phys. Rev. Lett. , vol. 105, p. 242001 , 2010.

[15] R. W. McAllister and R. Hofstadter, "Elastic Scattering of 188-Mev Electrons from the Proton and the Alpha Particle," Physical Review, vol. 102, no. 3, pp. 851-856, 1956.

[16] R. Hofstadter, "Nuclear and Nucleon Scattering of High-Energy Electrons," Ann. Rev. Nucl. Part. Sci., vol. 7, pp. 231-316, 1957.

[17] M. N. Rosenbluth, "High energy elastic scattering of electrons on protons," Physical Review, vol. 79, no. 4, pp. 615-619, 1950.

[18] J. Arrington and I. Sick, "Evaluation of the Proton Charge Radius from Electron-Proton Scattering," J. Phys. Chem. Ref. Data, vol. 44, no. 3, p. 031204, 2015.

[19] R. Karplus, A. Klein and J. Schwinger, Phys. Rev. , vol. 86, no. 288, 1952.

[20] J. L. Friar, "Nuclear Finite-Size Effects in Light Muonic Atoms," Annal of Physics, vol. 122, pp. 151-196, 1979.

[21] K. Pachucki,, Phys. Rev. , vol. A53, no. 2092, 1996.

[22] K. Pachucki, Phys. Rev. , vol. A 60, no. 3593, 1999.

[23] M. I. Eides, H. Grotch and V. A. Shelyuto, Phys. Rep, vol. 342, no. 63, 2001.

[24] U. D. Jentschura, Ann. Phys. , vol. 326, no. 500, 2011.

[25] E. Borie, "Lamb shift in light muonic atoms — Revisited," Ann. Phys, vol. 327, no. 3, pp. 733-763, 2012.

[26] A. Beyer, L. Maisenbacher, A. Matveev, R. Pohl, K. Khabarova, A. Grinin, T. Lamour, D. C. Yost, T. W. Hänsch, N. Kolachevsky and T. Udem, "The Rydberg constant and proton size from atomic hydrogen," Science, vol. 358, pp. 79-85, 2017.

[27] D. J. Berkeland, "Thesis, Yale University (1995).," 1995.

[28] M. Horbatsch and E. A. Hessels, Phys. Rev. A , vol. 82, p. 0525, 2010.

[29] A. Marsman, M. Horbatsch and E. A. Hessels, J. Phys. Chem. Ref. Data, vol. 44, p. 031207 , 2015.

[30] P. J. Mohr, B. N. Taylor and D. B. Newell, "CODATA recommended values of thefundamental physical constants: 2006," Rev. Mod. Phys., vol. 80, pp. 633-730, 2008.

[31] R. Aaij et al. (LHCb Collaboration), 
"Measurement of the Ratio of Branching Fractions," Phys. Rev. Lett., vol. 115, p. 111803, 2015.

[32] N. Haramein, "Quantum Gravity and the Holographic Mass," Phys. Rev. Res. Int., vol. 3, pp. 270-292, 2013.

[33] N. Haramein, "Addendum to "Quantum Gravity and the Holographic Mass" in View of the 2013 Muonic Proton Charge Radius Measurement," https://doi.org/10.31219/osf.io/4uhwp, 2013.

[34] N. Haramein and A. K. F. Val Baker, "Resolving the vacuum catastrophe: a generalized holographic approach," Journal of High Energy Physics, Gravitation and Cosmology, vol. 5, pp. 412-424, 2019.

[35] A. K. F. Val Baker, N. Haramein and O. Alirol, "The electron and the holographic mass solution," Physics Essays, vol. 32, no. 2, pp. 255-262, 2019.

[36] https://physics.nist.gov/cgi-bin/cuu/Value?rp. 